\title{
Modular design of a gas mixing apparatus for complex trace gas mixtures
}

\author{
Dennis Arendes ${ }^{1}$, Tobias Baur ${ }^{2}$, Henrik Lensch ${ }^{1}$, Johannes Amann ${ }^{1}$, Andreas Schütze ${ }^{1}$ \\ ${ }^{1}$ Lab for Measurement Technology, Saarland University, Saarbrücken, Germany \\ $23 \mathrm{~S} \mathrm{GmbH}$, Saarbrücken, Germany \\ Contact:d.arendes@Imt.uni-saarland.de
}

\section{Introduction}

Volatile organic compounds (VOCs) found in our environment can lead to reduced cognitive functions or serious health problems at elevated concentrations [1]. Their sources are manifold and range from the human metabolism to human activities such as cooking, heating, and cleaning, to emissions from furnishings, hygiene articles and building materials [2]. With the gold standard gas chromatography-mass spectrometry (GC-MS) being too expensive for consumer applications and not real-time capable, cost effective real-time measurement of these substances is possible by using metal oxide semiconductor (MOS) gas sensors combined with dynamic operation [3]. However, to make full use of the increased selectivity, the MOS sensor systems must be calibrated with application-specific complex gas mixtures. For this calibration, a gas mixing apparatus (GMA) is needed to produce well-defined, adjustable, and reproducible gas mixtures. Since more than 300 different VOCs are found in indoor air [4], creating an exact replicate of indoor air or similar complex atmospheres is not feasible. This contribution describes the design and realization of a GMA being able to provide sufficiently complex and representative, yet still manageable VOCs mixtures also with realistic atmospheric background of humidity and inorganic gases.

\section{Design basics}

Basically, a GMA is based on a carrier gas flow, in this case zero-air, to which different gaseous substances are added via several test gas flows forming the measurement line flow. Zero-air is dry (dew point below $-35^{\circ} \mathrm{C}$ ), purified air which contains very low concentrations of trace gases (e.g., hydrogen and hydrocarbons with a concentration below $0.1 \mathrm{ppm}$ ) due to catalytic purification [5]. The required variable concentrations can be set by varying the volume flows of the test gas flows. The different volume flows are set by using mass flow controllers (MFCs). The MFCs used are from the GMA50A model series from the company MKS Instruments Deutschland $\mathrm{GmbH}$.

Therefore, a simple test gas generation is performed by a test gas cylinder with a defined gas concentration, typically magnitudes higher than needed, connected to an injection MFC which controls the substance's flow, followed by a valve (here type 6624 from the Bürkert $\mathrm{GmbH} \&$ Co. KG). An optional pressure sensor can be installed between gas cylinder and MFC to monitor the inlet pressure of the MFC. Hence, the dynamic range of gas concentrations is determined by gas cylinder concentration, injection flow, and measurement line flow. The smallest possible concentration is determined by the minimum flow rate of the MFC.

The purity of test gas cylinders is typically $99.999 \%$ (rating 5.0) meaning that impurities up to $10 \mathrm{ppm}$ are included. Due to that, there is a natural lower limit of available concentrations for gas cylinders. In order to generate ultra-low concentrations of a test gas a more complex set-up is needed. To lower the impact of impurities, a gas cylinder with a higher test gas concentration is used and diluted with zero-air prior to injection into the mixing chamber. The dilution stage evenly dilutes target gas and impurities.

The concept of this two-stage pre-dilution was already introduced in [6] and was only fluidically optimized in the new GMA. The dilution consists of a gas MFC with small flow rate (typically a maximum flow of 10 or $20 \mathrm{ml} / \mathrm{min}$ ) which has the test gas attached to it and a larger flow dilution MFC (500 $\mathrm{ml} / \mathrm{min}$ ) which is attached to zero-air. With these two MFCs it is possible to generate a dilution depending on the flow ratio of both MFCs. Finally, the prediluted mixture is injected into the measurement line with an injection MFC (same size as the gas MFC) and a valve as mentioned before. This module can also have the optional pressure sensor between gas MFC and cylinder as before, but an additional pressure sensor in the predilution mixing chamber is mandatory. The injection MFC needs a constant differential pressure to work properly and must therefore be monitored constantly, since this pressure is set by the flow setting of two MFCs, not automatically by the cylinder pressure (the necessary pressure varies for different MFC manufacturers but is set to 2 bar in our system). This intermediate pressure is controlled via a pressure regulator attached to the mixing stage, the output of which is connected to the exhaust line. In addition, a further valve has been installed upstream of the gas MFC, which applies either test gas or zero air to the inlet of the MFC, so that a line can be completely purged without consuming other test gas. This valve is type 0330 also from the Bürkert $\mathrm{GmbH} \& \mathrm{Co}$. KG.

Due to this predilution the GMA can achieve a dilution ratio of the cylinder concentration varying from 1:1 with no separate carrier gas flow in the measurement line up to $1: 12505000$ with a measurement line gas flow of $1000 \mathrm{ml} / \mathrm{min}$. The range of the dilution ratios depends on the size of the MFCs used. The values above were calculated based on a $20 \mathrm{ml} / \mathrm{min}$ injection MFC, a $500 \mathrm{ml} / \mathrm{min}$ dilution MFC and a $20 \mathrm{ml} / \mathrm{min}$ gas MFC. This allows a wide concentration range of test gases, which is needed to simulate indoor air conditions where VOCs typically occur mostly in the low ppb range, but also up to several ppm are found during cleaning or cooking. 
With this principle, it must be ensured that the prediluted gas is completely mixed. Depending on the flow behavior, there are different ways to mix gases. The turbulence behavior of flows is indicated by the Reynolds number $R e$ as a characteristic number. This indicates whether laminar or turbulent flows are formed. In pipelines, a Reynolds number of less than 2100 corresponds to laminar flow and a Reynolds number greater than 4000 to turbulent flow. The Reynolds number is given by

$$
\operatorname{Re}=\frac{\rho \cdot \overline{\mathrm{v}} \cdot \mathrm{l}^{*}}{\eta}
$$

where $\rho$ is the density of the medium, $\bar{v}$ is the mean velocity, $\eta$ is the viscosity, and $l^{*}$ is the characteristic length [7]. Assuming a cylindrical tube with a diameter of $1 / 16$ inch with a constant flow rate of $500 \mathrm{ml} / \mathrm{min}$ air, results in a mean flow velocity of approx. $13.8 \mathrm{~m} / \mathrm{s}$. With the density of air of $1.2041 \mathrm{~kg} / \mathrm{m}^{3}$ and $\mathrm{l}^{*}$ being the tube diameter, this results in a Reynolds number of 848 , hence, in laminar flow, in which a total mixing of gases is unlikely. Mixing of gases under laminar flow conditions is only caused by diffusion.

For this reason, a diffusion section with a defined length is included, built of a 1/16-inch mixing tube which is coiled to save assembly space. If the distance is too short, there will be insufficient mixing and the set concentration will be incorrect. If the diffusion distance is too long, the settling time of the line suffers, requiring more time than necessary to traverse the assembly, thereby increasing dead time between two different concentrations. The selected distance considers a small diffusion constant and therefore slowest mixing time required for complete diffusion. The following relationship generally applies:

$$
\mathrm{Q}=\frac{\mathrm{V}}{\mathrm{t}}=\frac{\mathrm{A} \cdot \mathrm{L}}{\mathrm{t}}=\frac{\pi \cdot \frac{\mathrm{d}^{2}}{4} \cdot \mathrm{L}}{\mathrm{t}}
$$

Here, $Q$ is the volume flow in the tube, $V$ the volume, $t$ the time, A corresponds to the tube cross-section, $L$ the tube length and $d$ the tube diameter. The average square distance $\left\langle x^{2}\right\rangle$ traveled is considered, the diffusion constant $D$ is described by the Einstein-Smoluchowski equation [8]:

$$
\mathrm{D}=\frac{\left.<\mathrm{x}^{2}\right\rangle}{2 \cdot \mathrm{t}}
$$

In the worst case, the considered average squared distance $\left\langle x^{2}>\right.$ becomes even to the square of the diameter $d$. By substituting these two equations into each other, the time can be shortened out of the equation and finally rearranged according to the length $L$.

$$
\mathrm{L}=\frac{2 \cdot \mathrm{Q}}{\pi \cdot \mathrm{D}}
$$

The diffusion constant was determined using ChapmanEnskog theory [9]:

$$
\mathrm{D}=\frac{3}{8} \cdot\left[\frac{\mathrm{N} \cdot\left(\mathrm{M}_{1}+\mathrm{M}_{2}\right)}{\pi \cdot 2 \cdot \mathrm{M}_{1} \cdot \mathrm{M}_{2}}\right]^{\frac{1}{2}} \cdot \frac{\left(\mathrm{k}_{\mathrm{B}} \cdot \mathrm{T}\right)^{\frac{3}{2}}}{\mathrm{p} \cdot \sigma_{12}^{2} \cdot \Omega}
$$

Here, $\mathrm{N}$ is the Avogadro constant, $\mathrm{k}_{\mathrm{B}}$ the Boltzmann constant, $\mathrm{T}$ the temperature in Kelvin, $\mathrm{p}$ the absolute system pressure, $M_{i}$ the molar masses of air and the gas diffusing through it, $\sigma$ the collision diameter and $\Omega$ the collision integral. The index 12 represents the arithmetic mean of the collision diameters of air and the diffusing gas. For the desired case, the following assumptions are made: an absolute pressure of 3 bar and a temperature of $293 \mathrm{~K}$. The test gases diffuse into dry zero-air. Its average molar mass $\mathrm{M}$ is $0.028949 \mathrm{~kg} / \mathrm{mol}$. The collision integral $\Omega$ is not always determined, but is of the order of slightly above one [9]. To consider the worst case, a value of 1.1 is assumed. The collision diameter of air in this case is $3.617 \cdot 10^{-10} \mathrm{~m}$ (average of all components). In the worst case, the diffusion constant is small. For instance, toluene with a diffusion diameter $\sigma$ of $1.2 \mathrm{~nm}$ and a molar mass of $92.14 \mathrm{~kg} / \mathrm{mol}$ [9] has a diffusion constant in air of $1.0002 \cdot 10^{-5} \mathrm{~m}^{2} / \mathrm{s}$ which is the smallest of all test gases considered in the present setup.

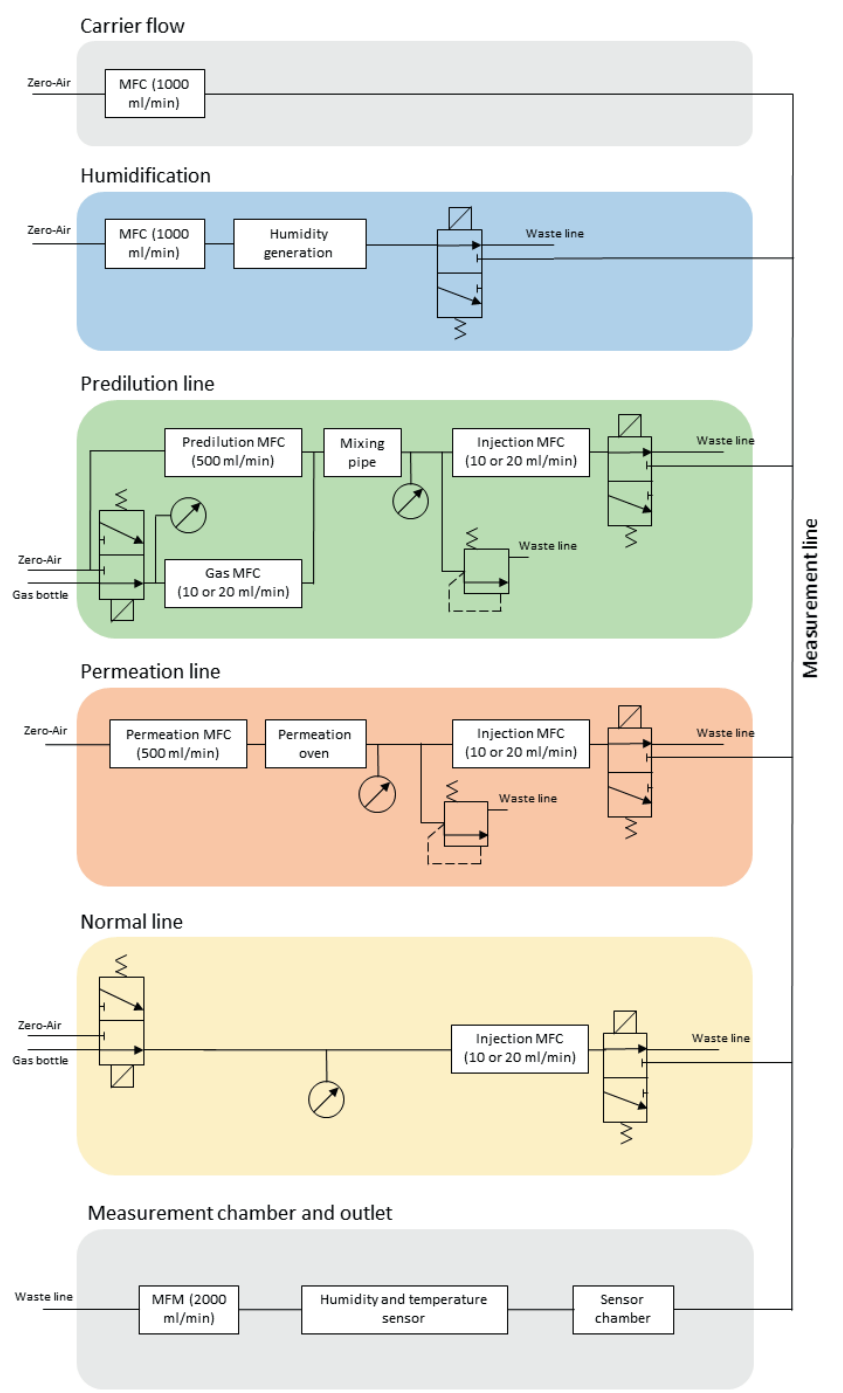

Figure 1 Schematic overall system of the GMA

The volume flow $Q$ in a line is just the maximum of the adjustable flows of the MFCs in the worst case. This is $520 \mathrm{ml} / \mathrm{min}$ for the selected MFCs. This results in a length of the mixing tube of $552 \mathrm{~mm}$. To allow an extension to other gases, a safety factor of 1.2 was selected, so that a tube with a length of $660 \mathrm{~mm}$ was installed. This also applies to the mixing of all gases in the measurement line. With a length of approximately $700 \mathrm{~mm}$ from the last injecting line to the actual apparatus outlet, this is sufficiently long 
for this purpose. For this reason, no additional mixing tube is required here.

For various reasons, especially low vapor pressure, it is sometimes not possible to acquire a substance in a gas cylinder. To bypass this problem for small concentrations another module, the permeation module, is designed. In this module, the test substance is generated by permeation from permeation tubes in permeation furnaces. An MFC is positioned in front of these furnaces, which sets a defined zero-air flow through the furnace. This enriches the zero-air with the permeated substance. The concentration of test gas after the permeation furnace can be further reduced by pre-dilution and then injected into the measurement line via the injection MFC as described before for the predilution line.

To achieve high concentrations of substances not available in gas cylinders (e.g., humidity, siloxanes) a bubbler module was created. Since humidity is the typical application for the bubbler module, due to the strong cross-sensitivity of MOS sensors to humidity [10], it serves as the example to describe the mode of operation. Humidity is defined as a mixture of gas and water vapor. It depends mainly on pressure, temperature, and the water vapor concentration. Humidity generation works via bubbling of zero-air through water in a wash bottle. In this process, a flow of zero-air is introduced through simple glass tubes or frits at the bottom of such a wash bottle and then bubbles upward through the respective liquid. The zero-air is thus humidified to saturation vapor pressure (100\% relative humidity). In order to suppress the impact of impurities (e.g. lime or organic impurities), water with the highest purity class, normally used for high performance liquid chromatography (HPLC), is used. This means that there are also few interfering substances in the final test gas. To filter out large water particles and aerosols, a second wash bottle is used in series to the first one, in which analytical grade glass wool is placed. This serves as a particle trap preventing these aerosols from reaching the measurement line and evaporating further after the humidity has been changed, thus falsifying the set humidity. Furthermore, sensors in the downstream could be wetted by droplets either disturbed the sensor operation or even destroying them. As humidity generation is temperature-dependent, as described above, both wash bottles are kept at a constant temperature of $20^{\circ} \mathrm{C}$ in a thermostat (Kiss K12 from Huber Kältemaschinenbau AG is used in the present GMA).

In order to also be able to measure at high humidity levels, e.g., $90 \% \mathrm{RH}$ or more, the total flow rate in the measurement line should not be too high. For a higher total flow in the measurement line at the same humidity, the flow through the wash bottles would have to be increased. However, above a certain flow speed, the humidified air in the bubbler no longer reaches the saturation vapor pressure because it is exchanged to quickly [11]. As a result, the actual humidity at the sensor no longer corresponds to the setpoint. To prevent this, the total carrier gas flow is usually reduced, so that the humidity line alone can provide the entire carrier gas flow if required. To monitor the current humidity level, a humidity sensor can be placed at the end of

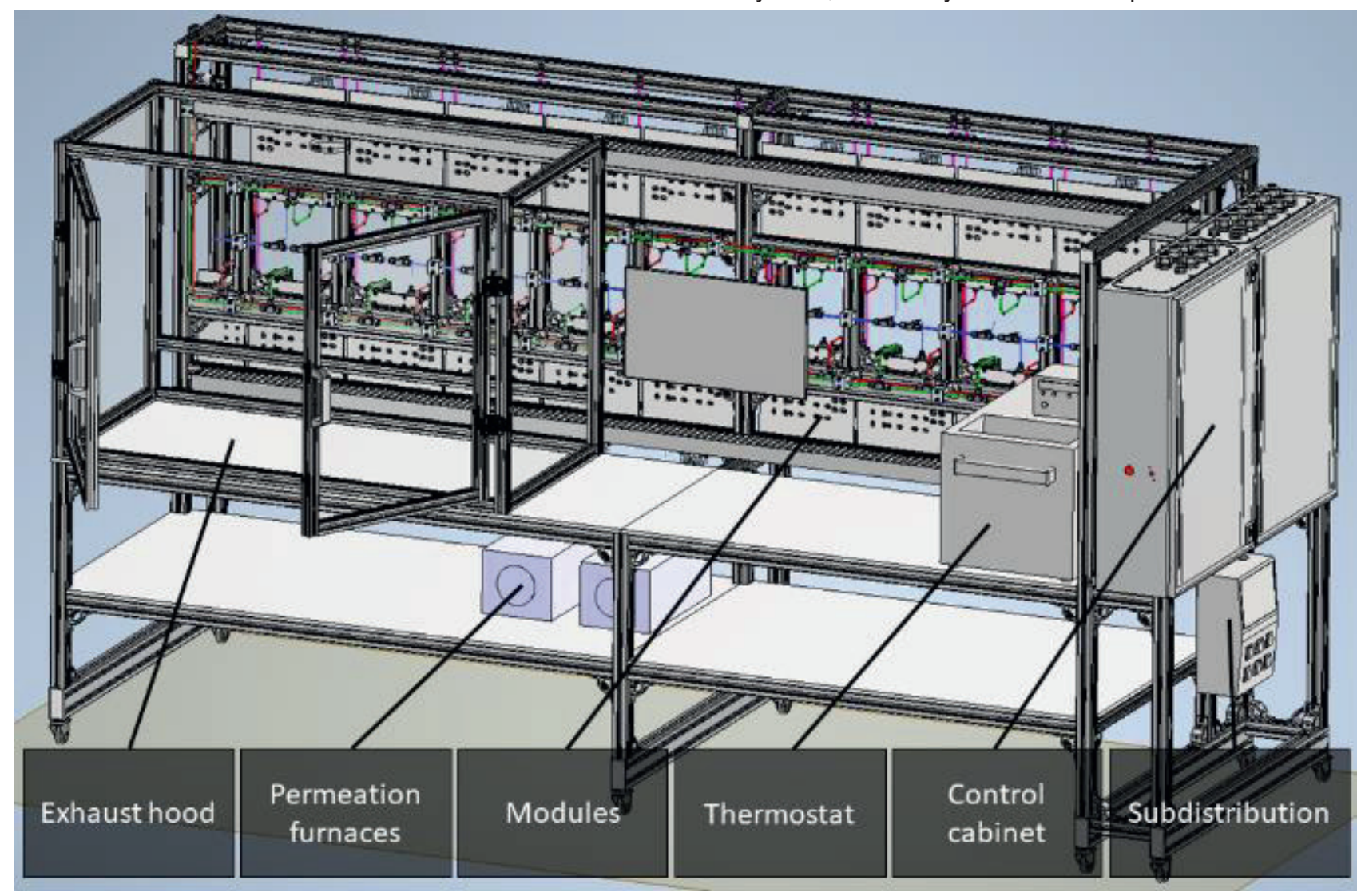

Figure 2 CAD model of the entire gas mixing apparatus from the operator side 
the measurement line. The overall GMA concept is shown in Figure 1. Mixing of all injected gases is achieved in the measurement line, which leads to the sensor chamber plus additional sensors for controlling the total flow, temperature and relative humidity.

\section{Concept realization}

The GMA was designed with a total of 21 independent gas supply lines, 18 of which are test gas lines. This allows measurement gas mixtures with one representative for each of the 12 relevant VOC substance groups [12] plus several interfering gases simultaneously. Each gas line can inject a specific gas into the shared carrier line, the resulting mixture is finally flowing to test chambers with the sensors to be calibrated. Great emphasis was placed on a standardized modular design allowing easy expansion and conversion. Based on this modular design, other methods of providing gases besides pre-dilution and permeation can also be integrated into the apparatus.

Three lines are mandatory for the basic function of the GMA. One carrier gas line and one humidity line, which set the desired total gas flow including the correct humidity, and one line with a mass flow meter (MFM) monitoring the total flow which can thus reveal leakage in the system or malfunction of individual MFCs. The remaining 18 lines can then be used as required by the modules described above.

The hardware is controlled by a programmable logic controller (PLC) receiving the control values for every device from a separate control computer. The control computer calculates the flow rate for each MFC and sets the corresponding valves for each gas exposure based on the gas concentrations set by the user. It also checks if the concentrations are within the limits of the dynamic range, monitors every feedback value, automatically detects failures (e.g., pressure failure, no or wrong MFC flow rate) and displays them in a GUI. The overall status of the apparatus is also

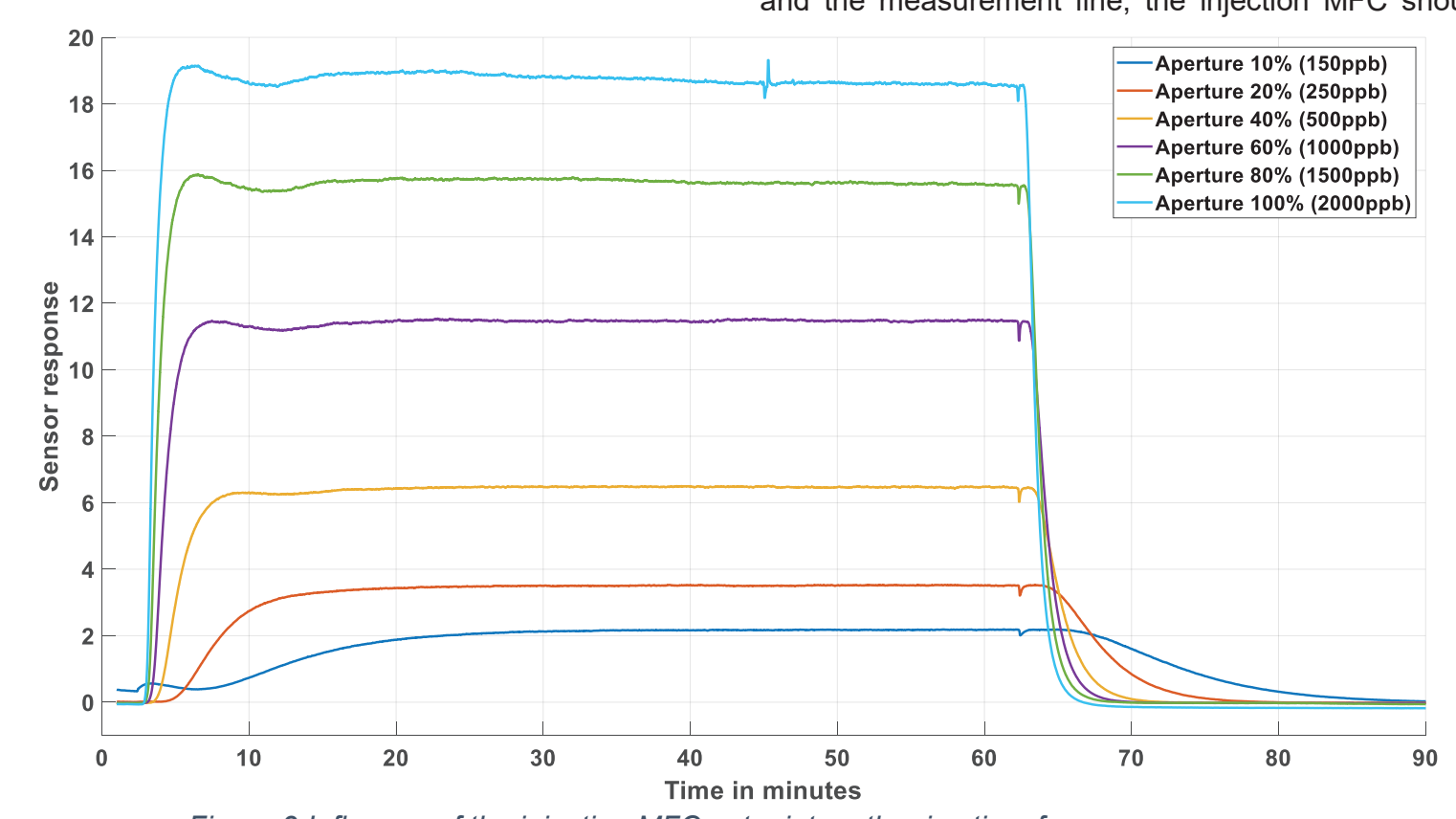

Figure 3 Influence of the injection MFC setpoint on the rise time for a gas exposure displayed via a signal tower. This control strategy is necessary since every exposure consists of up to 91 possible distinct values which must be set and monitored. Usually, one characterization measurement consists of several hundred gas exposures and is therefore prone to error if done by hand.

In total, the GMA is $3 \mathrm{~m}$ long, $1 \mathrm{~m}$ wide and $1.6 \mathrm{~m}$ high. The dimensions are based on the available space in the laboratory. The CAD model of the GMA is shown in Figure 2. Green pipes represent the distribution of zero-air to all modules and red pipes indicate the exhaust line. The measurement line is marked in blue. Supply pipes to connect the gas cylinders are shown in magenta.

\section{Optimization}

In a line with more than one MFC (predilution line, permeation line), identical concentrations can be achieved by different MFC flow settings. These settings have an influence on the GMA performance. This includes the test gas consumption, flush duration between two different concentrations and whether the predilution line has to be used with a closed predilution MFC passing the gas from gas cylinder to injection MFC without dilution.

These MFC setpoint options result in various states that must be avoided. Since some of these states are directly derived from the different performance options, conflicts of objectives arise. The software configuration for the desired setup handles the conflicting goals.

One undesirable state is a maximum opening of the gas MFC while the dilution MFC has not yet been minimized, resulting in unnecessarily high gas consumption. However, measurements have shown that a high gas MFC flow rate is beneficial for a fast concentration change within a line. The conflict of objectives that arises here is solved in the current software version by minimizing the gas consumption. Due to a large dead volume between the injection MFC and the measurement line, the injection MFC should be 
opened as much as possible. This increases the flow through the large dead volume and reduces the time between concentration changes.

At high humidity levels, another undesirable condition becomes apparent. The gases injected into the measurement line through the predilution lines are dry, i.e., have a relative humidity of $0 \%$. With a total flow of usually $1000 \mathrm{ml} / \mathrm{min}$ and a desired humidity of $90 \%$, a maximum of $100 \mathrm{ml} / \mathrm{min}$ can therefore be contributed from all 18 lines. Thus, if one injection MFC is then opened too much, gases from all available lines cannot be supplied, significantly restricting the dynamic range. Finally, the injection MFC cannot be fully opened in no-dilution mode since a relative pressure of 2 bar is required which is not possible without dilution and maximum opening of both MFCs.

To investigate these states and test various optimization rules, measurements were carried out on a single predilution line. The risetime of a predefined hydrogen concentration with different MFC settings was investigated. Hydrogen was used because it interacts well with the sensor used and at the same time is non-toxic to the user. The gas MFC as well as the injection MFC have a maximum flow rate of $20 \mathrm{ml} / \mathrm{min}$, the dilution MFC of $500 \mathrm{ml} / \mathrm{min}$. A SGP30 MOS sensor from Sensirion [13] was used for evaluation, operated at a constant temperature of $400{ }^{\circ} \mathrm{C}$. The second sensor layer was evaluated because it exhibits the lowest noise level. The sampling rate was $20 \mathrm{~Hz}$.

First, the influence of the injection MFC is examined in more detail. For this purpose, the gas MFC was opened to the maximum and the dilution MFC was varied to achieve a whole number as concentration as output. One gas exposure required $60 \mathrm{~min}$ with $30 \mathrm{~min}$ flush time with zero-air between two concentrations. The injection MFC was varied in 6 steps between $10 \%$ and $100 \%$. The results are shown in Figure 3.

Especially for very small injection MFC flow rates, the influence of the injection MFC on the resulting time constant is immediately obvious. In both the rising and falling slopes, a significant improvement is evident for larger injection flow rates with maximum injection flow achieving the smallest time constant. The result can be explained by the rather large dead volume leading to the injection MFC which is only flushed by the relatively small flow of the injection MFC with a maximum of $20 \mathrm{ml} / \mathrm{min}$. Therefore, it is advantageous to increase the flow rate of the injection MFC first before adjusting the two other MFCs for gas and predilution. Accordingly, further measurements were performed with the injection MFC set to maximum flow. Note that the small peak in the measurement with $100 \%$ opening after approx. $45 \mathrm{~min}$ is an artifact and has no further significance relevant for the evaluation.

Further measurements showed that the degree of opening of the dilution MFC has no relevant influence on the time constant. The degree of opening of the gas MFC, on the other hand, does. For this purpose, the injection MFC was opened to the maximum and the dilution MFC was set to $5 \%$ flow rate. The flow rate of the gas MFC was varied as shown in Figure 4.

Especially at the rising edge the influence of the gas MFC setpoint on the time constant is clearly shown. A large flow rate of the gas MFC is therefore favorable for optimization. However, this creates a conflict of objectives between the small time constant and the general desire to save (test) gas during measurements to reduce cost.

The reason for this effect of the MFC setpoint at first glance seems inexplicable, we suspect that this is caused by the test gas used, i.e., hydrogen. Therefore, the same measurement is repeated for acetone to investigate the influence of the gas on the time constant. The settings of all MFCs remain the same. The measurement shows the same, slower behavior for small gas MFC flow rates for acetone as for hydrogen. The cause of this effect is therefore not the used test gas.

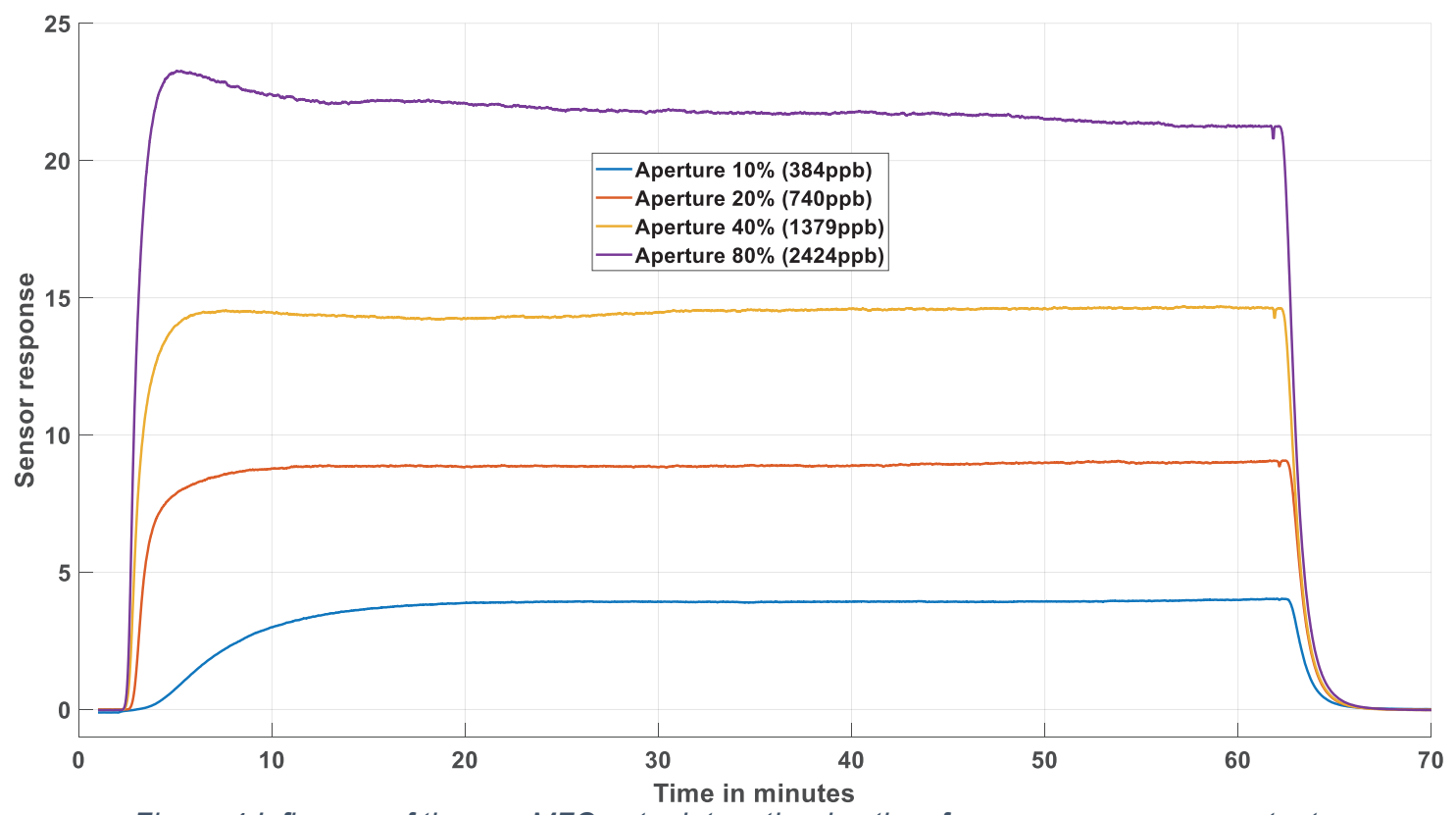

Figure 4 Influence of the gas MFC setpoint on the rise time for a gas exposure constant 
One possible cause for the observed increased time constant could be the dead volume between the gas MFC and the T-piece on the dilution MFC connecting both flows before the mixing section. During the set purge time of $30 \mathrm{~min}$, gas can diffuse from the gas MFC into the flow of the dilution MFC to a high degree, reducing the effective concentration in this area. Thus, when the next gas exposure starts the volume must first be flushed again with the higher cylinder gas concentration. Small flow rates in this area, determined by the gas MFC setpoint, therefore result in a longer duration until the concentration reaches its stable target value.

In summary, the GMA has the smallest time constants and allows fastest measurements when both the injection MFC and the gas MFC are opened as much as possible. However, this results in a target conflict for the gas MFC setpoint, where the low time constants and low gas consumption being mutually exclusive. The control software therefore sets concentrations mainly with the dilution MFCs, while the other two MFCs are opened to the maximum flow rates possible. These should be reduced only for small concentrations. The optimization rules can be adapted, e.g., for particularly expensive gases by opening the gas MFC as little as possible. This means, however, that longer adjustment times have to be accepted.

\section{Conclusion and outlook}

Due to the presented planning and realization, the new GMA is able to provide 18 different and independent test gases down to the sub-ppb range with humidity and total flow also being variable to allow different experimental protocols. Due to the modular design of the individual lines, the GMA can be extended as required and individual lines can easily be replaced by new gas generation methods, for example permeation or evaporation of liquids. The system operates completely autonomously based on an arbitrary measurement sequence. The MFC setpoints are calculated and adjusted automatically by the control software based on the selected gas concentrations. The apparatus has been optimized regarding speed during gas and concentration changes. These findings can also be used in existing GMAs to allow more measurements in the same time due to a faster setting time, which can reduce operating costs.

The new GMA is used in projects where up to 18 different independent test gases are required. These gases can be offered in any concentration range, from sub-ppb to higher ppm, with the predilution lines, allowing a wide dynamic range for many different gases. In addition, the system is easy to operate due to the software used, as the user can directly enter the required concentrations into the GUI of the software and thus manage large measurement series without manual calculations or intervention. Together with the optimization, a cost-efficient GMA was constructed, allowing improved gas sensor systems, e.g., based on randomized gas mixtures for indoor air quality monitoring [14, 15].

\section{Literature}

[1] Mølhave L., Nielsen G. D.: "Interpretation and Limitations of the Concept "Total Volatile Organic Compounds" (TVOC) as an Indicator of Human Responses to Exposures of Volatile Organic Compounds (VOC) in Indoor Air.", Indoor Air, 2009

[2] Belazzi T.: „Datenbankgestütztes Chemikalienmanagement zur Minimierung der VOC-Belastung der Innenraumluft", Tagungsband "Gesunde Raumluft Schadstoffe in Innenräumen "Prävention und Sanierung“, S. 52, Wien, 12.-13.2.2004 ISBN 3-900 40334-1

[3] Schütze A., Sauerwald T.: „Dynamic operation of semiconductor sensors," in: Jaaniso R. \& Ooi Kiang T. (eds.): Semiconductor Gas Sensors, Woodhead Publishing, 2nd Edition, 2020, Pages 385-412.

[4] Hofmann H., Plieninger P.: „Bereitstellung einer Datenbank zum Vorkommen von flüchtigen organischen Verbindungen in der Raumluft", WaBoLuHefte, 2008, 5, 161

[5] VICI DBS S.r.I.: „Product Brochure GC PLUS Zero-air Generator", checked November 2021

[6] Helwig N., Schüler M., Bur C., Schütze A., Sauerwald T.: „Gas mixing apparatus for automated gas sensor characterization," Meas. Sci. Technol. 25 (2014) 055903, doi: 10.1088/0957-0233/25/5/055903

[7] Denn M., „Process Fluid Mechanics,“ Prentice-Hall, 1980, ISBN: 978-0137231638

[8] Vogel G., "Diffusion und Brownsche Bewegung: Analogien bei der Wanderung von Atomen, Tieren, Menschen und Ideen," in Kärger, J.: "Leipzig, Einstein, Diffusion“ von Leipziger Univ.-Verl., 2014, ISBN: 9783-86583-176-7

[9] Hirschfelder J. O., Curtiss C. F., Bird, R. B.: "Molecular theory of gases and liquids" John Wiley \& Sons Inc., 1964, ISBN: 0471400653, Page 1145 \& $1246 \mathrm{f}$.

[10] Oehme F.: „Chemische Sensoren heute und morgen, Anforderungen, Fertigungstechniken, Entwicklungstrend", Die deutsche Bibliothek, 1994, ISBN 3-81690959-0

[11] Stucky M.: „Entwurf, Aufbau und Erprobung eines Klimaprüfstandes für Gassensoren“, Diplomarbeit, Lehrstuhl für Messtechnik, Universität des Saarlandes, Saarbrücken, 2002

[12] VDI/VDE-Gesellschaft Mess- und Automatisierungstechnik (GMA): „Multigas sensors - Standardized test instructions and test gases for VOC detectors for indoor air quality measurement", Richtlinie 3518 Blatt 4, https://www.vdi.de/richtlinien/details/vdivde-3518blatt-4-multigassensoren-standardisierte-pruefanweisung-und-pruefgase-fuer-voc-detektoren-zur-innenraumluftguetemessung, Aufruf am 22.10.2021

[13] Sensirion AG: „Datenblatt SGP30“, Mai 2020

[14] Baur T., Bastuck M., Schultealbert C., Sauerwald T., Schütze A.: "Random gas mixtures for efficient gas sensor calibration," J. Sens. Sens. Syst. (2020) 9, 411-424, doi: 10.5194/jsss-9-411-2020

[15] Baur T., Amann J., Schultealbert C., Schütze A.: "Field Study of Metal Oxide Semiconductor Gas Sensors in Temperature Cycled Operation for Selective VOC Monitoring in Indoor Air" Atmosphere (2021) 12, 647, doi 10.3390/atmos 12050647 